\title{
Clinical Detection of Primary Pulmonary Angiosarcoma
}

\author{
Kaynat Khalid ${ }^{1}$, Anas Khan ${ }^{1}$, Christine M. Lomiguen ${ }^{2,3}$, Justin Chin ${ }^{2,4}$ \\ 1. Department of Primary Care, Lake Erie College of Osteopathic Medicine, Erie, USA 2. Department of Medical \\ Education, Lake Erie College of Osteopathic Medicine, Erie, USA 3. Department of Family Medicine, Millcreek \\ Community Hospital, Erie, USA 4. Department of Family Medicine, LifeLong Medical Care, Richmond, USA
}

Corresponding author: Justin Chin, justinchindo@gmail.com

\begin{abstract}
Pulmonary angiosarcomas (PAS) are rare malignant vascular tumors that due to their aggressive and metastatic nature, are often diagnosed at a late stage, resulting in a poorer prognosis. Here we present a 53year-old male who was initially found to have recurring episodes of dyspnea and syncope, with initial workup showing bilateral saddle pulmonary embolisms on computerized tomography, presumed secondary to newly discovered atrial fibrillation with sinus node dysfunction. Further investigation over subsequent months and subsequent biopsy of a potential lung mass ultimately revealed pulmonary angiosarcoma of the spindle cell line. This case emphasizes findings in the current literature, which reveal the time between the onset of symptoms and definitive diagnosis ranges from two to six months, with a median survival time of seven months or two months, with solitary lesions or multiple lesions at the time of diagnosis, respectively. With the limited incidence of PAS, this case suggests benefits in the development of screening and detection criteria for earlier detection and treatment.
\end{abstract}

Categories: Family/General Practice, Oncology, Pulmonology

Keywords: pulmonary angiosarcoma, cancer, saddle pulmonary embolism, atrial fibrillation, syncope, pulmonary tumor, metastatic cancer, vascular tumor, primary angiosarcoma, pas

\section{Introduction}

Angiosarcomas are malignant endothelial neoplasms that commonly affect the skin, soft tissue/fascia, breast, and liver. Due to their aggressive and metastatic nature, angiosarcomas are often diagnosed at a late stage, resulting in a poorer prognosis [1]. First described in 1923 after reclassification of a primary cardiac tumor, pulmonary angiosarcomas (PAS) are classically found on the pulmonary arteries [2,3]. Subsequent literature and studies have been sparse, as PAS is frequently misdiagnosed due to a lack of defining symptoms as well as symptoms that more closely resemble more common diseases or conditions, such as thromboembolism $[4,5]$. Nevertheless, early detection and diagnosis have been reported, allowing for prompt resection of the tumor and increased odds of remission [6,7]. Due to the lack of proper diagnostic criteria, PAS is often misdiagnosed or underdiagnosed, leading to progression of the disease and high mortality $[8,9]$.

Received 07/24/2021

07/26/2021 Review ended 07/29/2021 Published 08/10/2021

\section{() Copyright 2021}

Khalid et al. This is an open access article distributed under the terms of the Creative Commons Attribution License CC-BY 4.0., which permits unrestricted use, distribution, and reproduction in any medium, provided the original author and source are credited.
Here we present a case of PAS in a 53-year-old male with recurring episodes of dyspnea and syncope that resulted in bilateral pulmonary embolisms. Common presentations and literature of PAS are also reviewed.

\section{Case Presentation}

A 53-year-old Middle Eastern male with no formal past medical or surgical history presented to the emergency room in New York following a syncopal episode while walking at work. Prior to this, he had never experienced syncopal episodes or any pre-syncopal symptoms. On initial workup, the patient had bilateral saddle embolisms on computerized tomography, presumed secondary to a newly discovered atrial fibrillation with sinus node dysfunction. The patient denied episodes of palpitations or exertional chest pain prior to the discovery of atrial fibrillation. Cardiology and pulmonology services advised pacemaker versus implantable cardioversion defibrillator placement with appropriate anticoagulation and thrombolytics. While awaiting treatment, a cardiothoracic service was consulted for a second opinion for the concern of thoracic malignancy as the primary cause.

The patient was transferred to a tertiary care center for further testing and biopsy over the next two months, which ultimately revealed pulmonary angiosarcoma of the spindle cell line. The patient was started on a course of ifosfamide and adriamycin and frequently returned for treatment and sequelae associated with pulmonary hypertension and symptom progression. Due to the large size, poor prognosis, and spread of his mass, he was not a candidate for resection, with eventual enlargement and localized spread. In the following six to eight months, the patient attempted naturopathic and complementary therapies, which included herbal, spiritual, and cultural remedies, in addition to his chemotherapy, with limited improvement in symptoms. 


\section{Cureus}

One year after his initial diagnosis, the patient passed away on palliative pain management after an episode of dyspnea and chest pain.

\section{Discussion}

Primary pulmonary angiosarcoma is an aggressive and rare form of pulmonary artery malignancy. Secondary pulmonary angiosarcoma, in which metastasis to the lung is from another primary site such as the heart or breast, is more common [10]. Similar to other angiosarcomas, PAS is derived from vascular endothelial cells [1,2]. To this date, less than 30 cases have been reported and described in the literature, resulting in an incidence rate of less than $0.030 \%$ of the general population (Figure 1) [11]. While cases of PAS have been found predominantly in middle-aged males, very little is known about its clinical course, prognosis, or other disease predisposition due to its rarity $[12,13]$. Currently, there are no established criteria to screen or triage cases that resemble PAS as its rarity precludes its inclusion in the differential diagnosis of common healthcare providers.

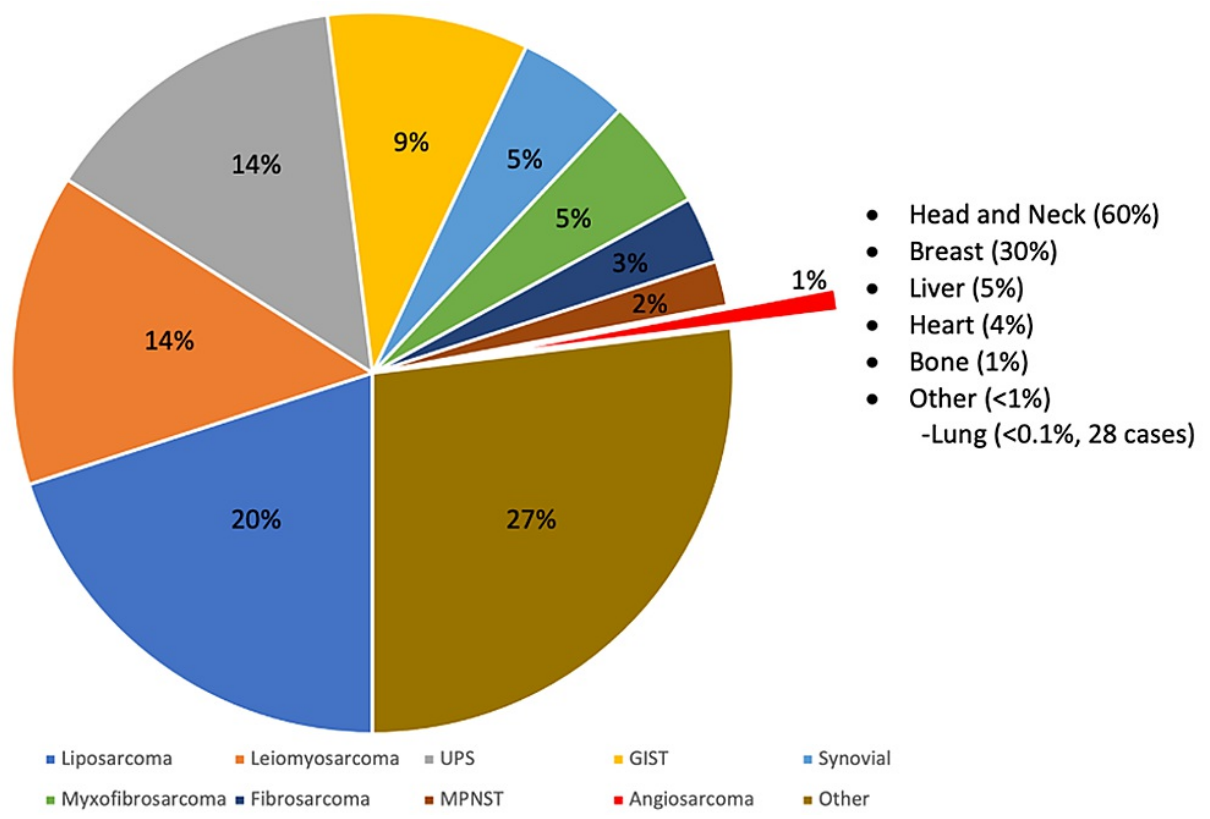

\section{FIGURE 1: Relative distribution of soft tissue sarcomas in the adult population. Angiosarcomas comprise $1 \%$ of cases, with head/neck and breast being the most common. Primary pulmonary angiosarcomas make up $<0.1 \%$ of cases.}

UPS = undifferentiated pleomorphic sarcoma; MPNST = malignant peripheral nerve sheath tumor; GIST = gastrointestinal stromal tumor. Adapted from the data published by Memorial Sloan Kettering Cancer Center [11].

As seen in previous reports of PAS, one of the largest obstacles to treatment is prompt detection. The average time between the presentation of initial symptoms to biopsy can range widely, from two to six months, resulting in delayed diagnosis, treatment, and overall care $[8,14]$. As seen in this case, although two months elapsed between initial hospitalization and biopsy, the patient had concerning symptoms for over a year. Misdiagnosis is common in PAS as symptoms are nonspecific and mimic other more common disease processes [15]. Nonspecific constitutional and respiratory symptoms can include weight loss, shortness of breath, cough, hemoptysis, and chest pain, for which the differential diagnoses encompass more common diseases in various organ systems. In the case presentation, the bilateral saddle embolisms were attributed to the new-onset atrial fibrillation rather than the hypercoagulable state of PAS. Exertional dyspnea and syncope are prevalent symptoms in many cardiovascular pathologies, including PAS. Due to lack of diagnostic criteria, recognition and subsequent treatment of PAS was delayed in this case, resulting in rapid disease progression that precluded the possibility of remission or surgical intervention [16]. Current treatments are limited but tend to be more effective in earlier stages [14,16].

Clinical suspicion is key in the early identification of PAS. Past medical history and the presence or lack of risk factors in more common diseases are important when investigating non-specific symptoms. Many of the related case reports in the literature note a lack of smoking or tobacco use, which is common in other lung cancer/pathology workups [17]. By doing so, healthcare providers are better able to question the working diagnosis when it does not fit well with the clinical history. In the aforementioned case, the patient did not 
have any prior cardiovascular disease or traditional risk factors such as smoking, obesity, or atherosclerosis. His acute symptoms should have warranted greater scrutiny in the context of overall health such that new atrial fibrillation would likely not be the primary cause of bilateral saddle pulmonary embolisms. Physicians must remain vigilant and detail-oriented when assessing patients with cardiovascular symptoms such as the ones described in this case report [18-20]. Further studies are needed to better screen and understand the disease progression for PAS.

\section{Conclusions}

In summary, primary pulmonary angiosarcoma is an exceedingly rare malignant vascular tumor with a nonspecific yet aggressive clinical course. As it stands, the time between the onset of symptoms and definitive diagnosis ranges from two to six months. Treatment tends to be more effective in earlier stages of primary PAS. It is highly suggested that screening and detection criteria allowing for earlier treatment of PAS be developed.

\section{Additional Information \\ Disclosures}

Human subjects: Consent was obtained or waived by all participants in this study. Conflicts of interest: In compliance with the ICMJE uniform disclosure form, all authors declare the following: Payment/services info: All authors have declared that no financial support was received from any organization for the submitted work. Financial relationships: All authors have declared that they have no financial relationships at present or within the previous three years with any organizations that might have an interest in the submitted work. Other relationships: All authors have declared that there are no other relationships or activities that could appear to have influenced the submitted work.

\section{References}

1. Obeso Carillo GA, García Fontán EM, Cañizares Carretero MÁ, Pérez Pedrosa A: Primary pulmonary angiosarcoma, an exceptional neoplasm with a poor prognosis: reports of two cases and review of the literature. Gen Thorac Cardiovasc Surg. 2013, 61:643-7. 10.1007/s11748-012-0200-6

2. Jain G, Mukhopadhyay S, Kurien S, Yusuf J, Tyagi S, Jain R: Ruptured cardiac angiosarcoma with pulmonary metastases: a rare disease with a common (mis)diagnosis!. Indian Heart J. 2012, 64:603-6. 10.1016/j.ihj.2012.07.016

3. Zwaveling JH, van Berkhout FT, Haneveld GT: Angiosarcoma of the heart presenting as pulmonary disease . Chest. 1988, 94:216-8. 10.1378/chest.94.1.216

4. Ferreira JS, Moreira N, Ferreira MJ, Antunes M: Angiosarcoma arising from the main pulmonary artery mimicking pulmonary thromboembolism. Arq Bras Cardiol. 2017, 109:90-1. 10.5935/abc.20170077

5. Kim JB, Kim SH, Lim SY, et al.: Primary angiosarcoma of the pulmonary trunk mimicking pulmonary thromboembolism. Echocardiography. 2010, 27:E23-6. 10.1111/j.1540-8175.2009.01059.x

6. Shirey L, Coombs D, Talwar A, Mickus T: Pulmonary epithelioid angiosarcoma responsive to chemotherapy: a case report. Radiol Case Rep. 2018, 13:479-84. 10.1016/j.radcr.2018.02.002

7. Atasoy C, Fitoz S, Yigit H, Atasoy P, Erden I, Akyar S: Radiographic, CT, and MRI findings in primary pulmonary angiosarcoma. Clin Imaging. 2001, 25:337-40. 10.1016/s0899-7071(01)00302-3

8. Correale M, Tarantino N, Ieva R, et al.: Don't stop at first glance: pulmonary artery angiosarcoma mimicking chronic thromboembolic pulmonary hypertension. Pulm Circ. 2018, 8:2045894018785047. $10.1177 / 2045894018785047$

9. Levy E, Korach A, Amir G, Milgalter E: Undifferentiated sarcoma of the pulmonary artery mimicking pulmonary thromboembolic disease. Heart Lung Circ. 2006, 15:62-3. 10.1016/j.hlc.2005.06.009

10. Yogi A, Miyara T, Ogawa K, Iraha S, Matori S, Haranaga S, Murayama S: Pulmonary metastases from angiosarcoma: a spectrum of CT findings. Acta Radiol. 2016, 57:41-6. 10.1177/0284185115571789

11. Brennan MF, Antonescu CR, Moraco N, Singer S: Lessons learned from the study of 10,000 patients with soft tissue sarcoma. Ann Surg. 2014, 260:416-21. 10.1097/SLA.0000000000000869

12. Ren Y, Zhu M, Liu Y, Diao X, Zhang Y: Primary pulmonary angiosarcoma: three case reports and literature review. Thorac Cancer. 2016, 7:607-13. 10.1111/1759-7714.12376

13. Ott RA, Eugene J, Kollin J, Kanas RJ, Conston DE, Chi JC: Primary pulmonary angiosarcoma associated with multiple synchronous neoplasms. J Surg Oncol. 1987, 35:269-76. 10.1002/jso.2930350413

14. Wilson R, Glaros S, Brown RK, Michael C, Reisman D: Complete radiographic response of primary pulmonary angiosarcomas following gemcitabine and taxotere. Lung Cancer. 2008, 61:131-6. 10.1016/j.lungcan.2007.12.006

15. Yu Y, Wang Q, Sun J, Zhao J, Chen S, Li Y: Fast growing angiosarcoma of the right atrium after radiofrequency catheter ablation: a missed diagnosis or misdiagnosis case report. BMC Cancer. 2020, 20:13. 10.1186/s12885-019-6450-2

16. Kojima K, Okamoto I, Ushijima S, Yoshinaga T, Kitaoka M, Suga M, Sasaki Y: Successful treatment of primary pulmonary angiosarcoma. Chest. 2003, 124:2397-400. 10.1378/chest.124.6.2397

17. Hsing JM, Thakkar SG, Borden EC, Budd GT: Intimal pulmonary artery sarcoma presenting as dyspnea: case report. Int Semin Surg Oncol. 2007, 4:14. 10.1186/1477-7800-4-14

18. Chen JH, Kaufman DI, Chin J, Lomiguen CM: Li-Fraumeni syndrome: adopting a diagnosis with an unknown family history. Am J Cancer Case Rep. 2019, 7:40-4.

19. Zhou Arlene Y, Chin J, Strutin MD, Lomiguen CM: Unmasking dermatofibrosarcoma protuberans: case report of an atypical presentation complicated by post-surgical excision. Int J Surg Case Rep. 2020, 69:101-4. 10.1016/j.ijscr.2020.03.020 


\section{Cureus}

20. Chin J, Migliozzi M, Nguyen G, Kviatkovsky B, Lomiguen C: Atrial invasion and showering phenomenon in primary lung cancer: a case report. Respir Med Case Rep. 2020, 30:101064. 10.1016/j.rmcr.2020.101064 